\title{
Assessing sensitivities of marine areas to stressors based on biological traits
}

Judi E Hewitt (corresponding author) j.hewitt@niwa.co.nz

Physical address- Gate 10 Silverdale Rd, National Institute of Water and Atmospheric Research, Hamilton, New Zealand 3216

Postal address NIWA, PO Box 11-115, Hamilton, New Zealand 3216

Docent at Department Marine Biology, University of Helsinki, P.O. Box 3 (Fabianinkatu 33), Finland 00014

Carolyn J Lundquist

Gate 10 Silverdale Rd, National Institute of Water and Atmospheric Research, Hamilton, New Zealand 3216

and Institute of Marine Sciences, The University of Auckland, 38 Princes St, Auckland, 1010

Joanne Ellis

King Abdullah University of Science and Technology, Thuwal 23955, Saudi Arabia

Running Head Assessing sensitivity using traits

Keywords: benthic species, risk assessment, anthropogenic activities, simulated impacts, extraction, sedimentation, suspended sediments

Article Impact Statement: Biological trait analysis can predict differences in sensitivity to different stressors enabling the prioritization of protected areas

\section{Abstract}

Analysis of the biological traits (BTA) that control how organisms interact with their environment has been used to identify environmental drivers or impacts across large-scales and to explain the importance of biodiversity loss. However, BTA could also be used within risk assessment frameworks or conservation planning by understanding the groups of traits that predict the

This article has been accepted for publication and undergone full peer review but has not been through the copyediting, typesetting, pagination and proofreading process, which may lead to differences between this version and the Version of Record. Please cite this article as doi: 10.1111/cobi.13181.

This article is protected by copyright. All rights reserved. 
sensitivity of observed habitats or communities to specific human activities. Deriving sensitivity from biological traits should extend sensitivity predictions to a variety of habitats, especially those in which it would be difficult to conduct experiments due to for example depth, risk to gear and human life and at scales larger than the normal scale of experiments. We used BTA on video transect data collected from a relatively pristine region of the seafloor to determine scales of natural spatial variability, the degree to which predictions of sensitivity are affected by underlying community compositions and the ability of the BTA to provide predictions that differ between three different stressors (extraction, sedimentation and suspended sediments). Three methods were used to assess sensitivity (weighted abundance, abundance of highly sensitive species and number of highly sensitive species). Regardless of method and spatial patterns occurring across the sampled area, BTA was able to distinguish differences in sensitivity at a site to different stressors. BTA also successfully separated differences in community composition from differences in sensitivity to stressors. Conversely, the three methods varied widely in their ability to detect simulated impacts. Differences between the methods reflected underlying processes, suggesting that use of multiple methods would be more informative for spatial planning and allocating conservation priorities than use of a single method. Our results suggest that BTA could be used as a first step in strategic prioritisation of protected areas and as an underlying layer for spatial planning.

\section{Introduction}

Species exhibit a range of biological traits (e.g., filter feeding, being highly mobile) that control their responses to their surrounding environment, their interactions with other species and their ability to affect their environment. Analyses of these traits (biological trait analysis; BTA) has a long history across many disciplines, with research on the use of BTA initially focusing on two fields. BTA has been used to allow comparisons across large-scales, where species pools change, such that environmental drivers or effects of disturbance can be determined (Bremner et al. 2006; McGill et al. 2006; Tillin et al. 2006; Menezes et al. 2010; de Juan \& Demestre 2012). It has also been used to 
explain the importance of biodiversity loss and in the development of biodiversity-ecosystem function research (Emmerson \& Raffaelli 2000; Bolam et al. 2002; Lavorel et al. 2011; Harvey et al. 2013; Strong et al. 2015) with functional diversity and redundancy being increasingly used concepts in recent ecological theories (Zhang et al. 2012; Ricotta \& Acosta 2014; Delgado-Baquerizo et al. 2016). Increasingly, it is used to assess not only whether an impact occurs but also the relative importance of that impact to ecosystem functioning (Oug et al. 2012). Conversely, BTA could be used within a risk assessment or conservation prioritisation framework to assess the sensitivity of habitats or areas to a planned activity (Zacharias \& Gregr 2005; Clark et al. 2016).

Vulnerability or sensitivity assessments are a formal process for assessing risk of activities to a system (Williams et al. 2008; Hare et al. 2016). Vulnerability generally incorporates the risk of the activity actually occurring (exposure) as well as the response of the system to the activity, which is usually defined as the sensitivity. While sensitivity has been assessed on purely physical terms (e.g., flushing (Ribeiro et al. 2016), or by expert elicitation (Hare et al. 2016), assessments can be improved by incorporating the traits that make a species sensitive to a specific stressor (Tyler-Walters et al. 2009). Vulnerability or sensitivity assessments have been used to evaluate risks associated with fishing (Hobday et al. 2011; Rijnsdorp et al. 2015) and climate change (Aubin et al. 2016; Hare et al. 2016). This approach fills the need for broad, transparent, relatively quick evaluation of the vulnerability of multiple species (Hare et al. 2016).

In order to use BTA to assess sensitivity, general characteristics that predict both the sensitivity to and the resistance to a stressor need to be known. In ecology, a range of study approaches are used to determine these characteristics from manipulative experiments, impact assessments and broadscale surveys. Generalities from individual or multiple studies have been used to inform the theory of disturbance/recovery and impact assessments. For example, Pearson \& Rosenberg (1978) characterised different stages of organic enrichment in marine systems based on sediment dwelling depth, burrowing activity, body size, mobility, and life span. Thrush et al. (1995) summarised the 
biological traits that would be affected by shellfish dredging and this was extended by Thrush \& Dayton (2002) to bottom fishing more generally.

Combining information on stressors, ecological studies and biological traits to assess sensitivity offers the potential to expand sensitivity analysis to a variety of habitats, especially those in which it would be difficult to conduct experiments due to for example depth, or risk to gear and human life. It also offers the ability to conduct risk assessments at scales well beyond the normal small scale of experiments. However, apart from the requirements for knowledge about the traits that confer sensitivity to a particular stressor, we also need to know whether this methodology is able to differentiate between different stressors and the degree to which the method would be affected by underlying community composition.

Difficulties in answering the above questions usually relate to the underlying fact that most areas have already been affected by one or more stressor and this would be expected to affect observed traits (Hiddink et al. 2007). Here, the potential sensitivities of communities are characterised in an area that could be considered pristine. The top of the Chatham Rise, New Zealand, is located approximately $500 \mathrm{~km}$ offshore and is relatively deep (300 - $500 \mathrm{~m}$ ). It is subject to low fishing effort due to bottom roughness limiting gear access; as yet no other extractive marine industries operate in the area. No experimental manipulations are used; rather BTA is used to derive potential sensitivity to three common global stressors arising from bottom fishing, mining, and maintenance of channels (i.e., extraction of organisms and/or sediments, sediment deposition, and suspended sediment content). While channel dredging and disposal of sediments is generally more common in shallow coastal areas, and more spatially and temporally restricted, fishing and mining occur from shallow areas to the deep ocean and can occur at large spatial and temporal scales.

Three different methods are used to derive the sensitivity of a transect from the sensitivities of the individual taxa: (1) weighted abundance summed across taxa (Stark \& Maxted 2007; de Juan et al. 2009); (2) the abundance (summed across all taxa) in the two most sensitive groups as a percent of 
the total abundance; and (3) the number of taxa in the two most sensitive groups (Hewitt et al. 2011). We had four objectives. Firstly, we tested two hypotheses: (1) that sites will differ in potential sensitivity to the different stressors; and (2) that different communities, identified across the study extent, will not necessarily differ in their sensitivities to a stressor, as similar biological traits may be present within differing communities. We then analysed (3) the patterns of spatial variability in trait sensitivity as this would be useful to inform scales for management and conservation. Finally, (4) we compared the detection ability of the three methods to detect stressors, anticipating that methods based on abundances rather than number of species would be more sensitive (Hewitt et al. 2005).

\section{Methods}

\section{Study sampling.}

Video images of the seafloor epibenthos were taken randomly from an area $300 \times 45 \mathrm{~km}$ that varied in depth from 340 to $480 \mathrm{~m}$ from 27 sites (Supporting Information Appendix S1). At 21 sites, 3 replicate transects were 'flown' by a remotely operated vehicle (ROV), containing a video camera, at a target height of $2 \mathrm{~m}$ above the seafloor and at a speed of 0.5 knot for approximately 1 nautical mile. The remaining sites were single transect sites used to extend the study area. The live video feed from the ROV was viewed on-board the research vessel by two biologists who logged their observations of benthic and pelagic organisms to the lowest taxonomic level, generally species/genus. The manipulator arms of the ROV were used to sample animals where the identification of particular taxa was uncertain. Counts were converted to numbers per $\mathrm{m}^{2}$.

\section{Assigning taxon to biological traits}

Expert taxonomists and ecologists provided information for the biological traits given in Table 1, for the majority of all identified taxa. Fuzzy coding was used to assess the relative probability of each taxa exhibiting the trait expression within each trait category, with the probability across the 
category summing to 1 . Where no knowledge specific to the taxon was available, trait probabilities were assigned in the following way: (1) if all known taxa within the higher taxonomic level exhibited the same traits, that taxon was given those trait probabilities; (2) if known taxa within the higher taxonomic level exhibited different traits, the taxon was given probabilities that reflected the distribution of known traits; and (3) if nothing was known about the traits, the taxon was assigned an equal probability of expressing all traits within the category.

\section{Assigning sensitivity to a taxon}

Here we dealt with direct sensitivity of an individual as we consider that this is best suited to the use of biological traits (de Juan \& Demestre 2012). We did not include ability to recover as part of sensitivity, rather we focused on resilience via survival through a disturbance (Thrush et al. 2009; Lambert et al. 2014; Hodgson et al. 2015). For each stressor, a decision tree was created that assigned combinations of trait expressions to five levels of sensitivity, sensu Tyler-Walters et al. (2009) (see Fig. 1). For extraction and suspended sediment, the five levels were high, medium and low sensitivity, neutral and benefit. For sedimentation, the levels were high, medium and low sensitivity, followed by less likely and least likely to be affected.

The probabilities of a taxa belonging to a particular level of sensitivity were calculated and ranged from 0 to 1 . For example, if in order to belong to the highest sensitivity a taxon had to exhibit the biological traits of "epibenthic" and "sedentary" then the probability of the taxon being epibenthic (as assigned by the previous section) was multiplied by the probability that it was sedentary (as assigned by the previous section) to give the probability that it was highly sensitive.

\section{Extraction}

Traits that were predicted to affect response to extraction were mobility, living position and feeding mode (see Thrush et al. 1995; Thrush \& Dayton 2002; de Juan et al. 2009; Clark et al. 2016 and references therein). Organisms living above the depth in the sediment to which sediment/organisms 
would be removed by gear (in this example set to $5 \mathrm{~cm}$ ) that were sedentary were assumed to be the most likely to be removed (highest sensitivity). Organisms living below that depth were assumed to be unaffected (neutral). Epibenthic organisms that are mobile enough to escape and feed on dead or broken organisms (i.e., are predators or scavengers) were assumed to benefit from the stressor (positive). Between this highest sensitivity and neutrality, mobility, living depth and feeding mode were assumed to control sensitivity (Fig. 1a), providing splits between "medium" and "low sensitivity" (five categories in total).

\section{Sedimentation}

Traits that were predicted to affect response to sedimentation were growth form, fragility, living position, mobility, size and feeding mode (see Thrush et al. 2004 and references therein; Boschen et al. 2016). Above seafloor growth form and fragility were used to place organisms into high, medium and low sensitivity categories with encrusting organisms being the most sensitive as they would be easily covered by sediment (Fig. 1b). Conversely, organisms that are not epibenthic and are subsurface deposit feeders were least likely to be sensitive. For sedimentation, the literature produced no general examples of traits that would benefit from increased sedimentation, so the five categories ranged from "highly sensitive "to "least likely to be affected".

\section{Suspended sediment}

Traits that were predicted to affect response to suspended sediments were living position, size and feeding mode (see Ellis et al. 2002; Thrush et al. 2004 and references therein). For example increased turbidity negatively effects suspension feeders, can increase the percentage of deposit feeders and negatively effects seagrass and seaweed communities that have higher requirements for sunlight (Ellis et al. 2002; Thrush et al. 2004). Therefore, plants or suspension feeding fauna were assumed to be the most sensitive (Fig. 1C). Predators living near the sediment surface were assumed to benefit from suspended sediment adversely affecting other organisms. 


\section{Methods for deriving transect level sensitivity}

We used three methods to derive a quantitative assessment of the sensitivity of a transect, from sensitivities of individual taxa. All three methods were based on the categorisation of the sensitivities into 5 levels and were conducted separately for each stressor. The first two methods were based on abundance while the third was based on the number of sensitive taxa.

(i) Weighted abundance. For each taxon, the probability of it exhibiting each sensitivity level (see Supporting Information Appendix S2) was multiplied by its average abundance per $\mathrm{m}^{2}$ and then by the weight of each sensitivity level. For extraction and suspended sediment, these weighting were high (3), medium (2), low (1), neutral (0) and benefit (1). For sedimentation, the weightings were high (4), medium (3), low (2), less likely to be affected (1) and least affected (0). These values were then summed over all taxa occurring in the transect.

(ii) Sensitive relative abundance. The probability of each taxa being of high or medium sensitivity was multiplied by its average abundance per $\mathrm{m}^{2}$. These values were summed over all taxa occurring in the transect and divided by the total abundance found on the transect (per $\mathrm{m}^{2}$ ).

(iii) Number of sensitive taxa. The number of taxa that had a greater than $0.5 \%$ probability of being highly sensitive (i.e., top two sensitive groups) was calculated.

\section{Statistical analyses}

Testing hypothesis 1: sites will differ in their calculated sensitivity to the different stressors

A Friedman's test (Proc freq, (SAS/STAT 2011)) was used to determine whether each site ranked the three stressors types in the same order. Weighted abundance, sensitive relative abundance and 
number of sensitive taxa values from the transects were averaged for each site and a separate test was done for each of the three methods. While technically three tests were run, we did not consider it necessary to correct for multiple tests as we were interested in the relative differences that we would have observed for the different methods had they been conducted in isolation.

Testing hypothesis 2: different communities do not differ in their potential sensitivities to a stressor.

Simprof analysis (PrimerE v7.09, Clark \& Gorley (2015)) was used to group the square root transformed transect species count data into different community groups across the area, using Bray-Curtis similarities, group average clustering and $p=0.01$. Twelve groups were identified (Table 2), with each transect belonging to one of these groups. Of these 12 groups, two had only one member and five had only two members and were removed from the subsequent hypothesis 2 analysis. For each method, a two-way general linear model including an interaction term (GLM; SAS/STAT 2011) with simprof group and stressor being fixed terms was used to determine whether there were differences between the groups based on type III tests. As the potential sensitivity of the stressors exhibited spatial autocorrelation across the study area (see objective 3), the error degrees of freedom were reduced to reflect the number of independent samples. Differences between simprof groups for each stressor were assessed by the degree of overlap of standard errors.

\section{Objective (3) Analysing patterns of spatial variability}

In order to gain an understanding of the scales of spatial variability, spatial autocorrelograms based on Moran's I, using equal intervals, were constructed in SAM (Rangel et al. 2010) for each stressor and method. The smallest distance interval used was $6 \mathrm{~km}$ as this allowed the transects from a site to be grouped together.

Objective (4) Comparison of detection ability of the three methods

To compare the ability of the three methods to detect changes that could result from the three stressors, nine simulation scenarios were developed based on reducing the abundance of taxa that This article is protected by copyright. All rights reserved. 
contributed to the two highest sensitivity levels. These were reductions of $10 \%, 25 \%, 40 \%, 50 \%$, $60 \%, 75 \%, 80 \%, 85 \%$ and $90 \%$ and were applied to the abundance of taxa that contributed to the two highest sensitivity levels separately for each of the three stressors calculated from all transects. These reductions can never result in a reduction to zero and thus can not result in changes to numbers of taxa. Therefore, when a change in abundance resulted in a taxon abundance below the minimum abundance found across all transects and taxa in the original data $\left(0.1\right.$ individuals per $\left.\mathrm{m}^{2}\right)$, the taxon abundance was set to zero, reducing the number of sensitive taxa. A series of t-tests (with a correction for unequal variances if required) were used to compare the original values to the scenario values for each stressor and method separately. As the degrees of freedom were the same for each t-test, the $p$-values could be used as an indication of the changing ability to detect the scenario, even though this is not the test that would be likely to be used to detect real impacts.

\section{Results}

Species observed in the transects varied from mobile taxa such as decapods (hermit crabs, shrimps and crabs) and echinoderms (starfish, urchins, holothurians) to more sedentary taxa such as sponges, bryozoans, corals and polychaetes (tube worms). They also varied in growth form from encrusting to erect with single or multiple branches. Average abundance per $\mathrm{m}^{2}$ across the sampled area ranged from 4 to 759 with an average of 121 individuals. Total number of taxa observed along a transect ranged from 2 to 27 with an average of 16 . Differences/similarities between the transects were driven by a small number of taxa (Appendix S3).

Testing hypothesis 1: sites will differ in their calculated sensitivity to the three stressors

Each stressor ranked the sites differently regardless of method ( $p<0.0001$ for each Friedman's test). All pairwise comparisons were significantly different, although for the sensitive relative abundance method the significance level was $p=0.0286$ for the extraction - suspended sediment comparison cf $<0.001$ for the other comparisons.

This article is protected by copyright. All rights reserved. 
Testing hypothesis 2: different communities do not differ in sensitivity to a stressor.

For all three methods, there was a significant interaction between stressor type and the community groupings identified by simprof (see Supporting Information Appendix S3 for descriptions of the groups), indicating that the different communities did vary in their sensitivities to the stressor type (stressor type*simprof group, d.f. $=8$, error d.f. $=8, p<0.02$ for all methods). For weighted abundance (Supporting Information Appendix S4a), group L was significantly higher than the other groups for all stressors. The other groups varied with stressor in the degree to which they differed from each other and whether group A or group B had the lowest mean. In terms of the sensitive relative abundance, group B followed by group A had the highest means for extraction, while group L and $\mathrm{A}$ had the highest means for both sedimentation and suspended sediment stressors (Supporting Information Appendix S4b). For the number of sensitive taxa (Supporting Information Appendix S4c), the means of the simprof groups ran in the same order for all stressors (L, D, J, A, B), but only for sedimentation was there a clear separation between groups. .

\section{Objective 3: Analysing patterns of spatial variability}

A significant Moran's I was observed for all methods and stressors $(p<0.05)$ at the site scale $(<6 \mathrm{~km})$. Significance of the autocorrelograms over the $160 \mathrm{~km}$ area were observed for all but weighted abundance of sedimentation and suspended sediment stressors. Regardless of stressor, autocorrelograms for sensitive relative abundance and number of sensitive taxa (see Supporting Information Appendix S5a) suggested an average spatial structure of $30 \mathrm{~km}$ (i.e., similarity of sensitivity declined away from sites and disappeared at approximately $30 \mathrm{~km}$ ). A similar, but nonsignificant, structure was observed for the weighted abundance method (see Supporting Information Appendix S5b).

This article is protected by copyright. All rights reserved. 
Objective 4: Comparison of detection ability of the three methods

The three methods exhibited different abilities to detect the simulated scenarios of reduced abundances caused by different stressors. For extraction, the sensitive relative abundance and weighted abundances had similar abilities, detecting reductions of $40 \%$ (and probably as low as $30 \%$ ), while the number of sensitive taxa could not detect any reduction less than $80 \%$ (Fig. 2). For sediment, the weighted abundance method could detect a reduction of at least $25 \%$, the sensitive relative abundance method could detect a reduction of $50 \%$, while the number of sensitive taxa again could not detect a reduction less than $80 \%$. For suspended sediment, the weighted abundance again detected the smallest reduction ( $35 \%)$, while the number of sensitive taxa method again could not detect a reduction less than $80 \%$. However, the sensitive relative abundance could not detect even a $90 \%$ reduction for suspended sediment.

\section{Discussion}

This study demonstrates that using biological traits to assess sensitivities of areas to stressors is a useful approach. The utility of BTA to assess stressors was consistently found, despite there being obvious shared spatial patterns across the area. Sites did differ in their calculated sensitivity to the different stressors (hypothesis 1), but not all communities differed in sensitivity (hypothesis 2). However, despite the three methods each giving the same results to the two hypotheses tested, the methods did vary widely in their ability to assess sensitivity to three types of benthic impacts. As predicted, methods based on abundance rather than taxa presence/absence were generally better able to detect impacts.

Hypotheses 1 and 2 are fundamental to the use of BTA in conservation planning and management. In order for the BTA approach to usefully assess sensitivity of an area or habitat, it needs to be able to produce scores that differ between stressors, yet not necessarily produce scores that differ between communities (i.e., not be too sensitive to differences in community composition). Achieving 
this requires that trait expressions that are sensitive to different stressors are not strongly correlated, and that a number of species share traits. The results from this study suggest that while there are likely to be species, such as small sponges, that are sensitive to all three stressors (extraction, sedimentation and suspended sediment), many other species are not (Sirot et al. 2015; Tolonen et al. 2016).

Spatial structure in the assessments of sensitivity was observed for the sensitive relative abundance and number of sensitive taxa methods for all three stressors. However, for the third method, weighted abundance, spatial structure was only observed for extraction. Similarity of spatial structure does not necessarily mean that any patches overlap in position, and indeed the differences between sites in sensitivity to the three stressors suggests that this is not the case. Regardless, the size of the spatial structures identified, together with the general lack of spatial structure identified for the weighted abundance method is useful information for converting sensitivity to vulnerability, where the spatial and temporal scale of the threat needs to be matched to the spatial and temporal scale of the area's sensitivity. Therefore, an assessment of the temporal dynamics of sensitivity would be an important future research question and could possibly be addressed using biological traits of generation time and adult mobility.

Deriving transect sensitivity revealed that the different methods (based on weighted abundance, sensitive relative abundance and number of sensitive taxa) had different abilities to predict and detect impacts. A previous study by Tyler-Walters et al. (2009) also observed differences in site sensitivity dependent on methods. They calculated site sensitivity from the distribution of species sensitivities as either the most frequent or the most sensitive, i.e., the mode or the maximum sensitivity, and observed that the mode consistently underestimated the total sensitivity of the community. Hewitt et al. (2011) observed that using the maximum sensitivity, whether it was calculated from the dominant species, or the species contributing most to within-site community similarity, resulted in a smoothing across habitats with nearly all habitats being designated as "highly 
sensitive". Hence this study choose to test the weighted abundance (Stark \& Maxted 2007; de Juan et al. 2009) and the number of sensitive taxa (Hewitt et al. 2011) methods. A method that removed differences in abundances between samples (sensitive relative abundance) in case these biased results and increased variance was also included.

Differences in ability of the three methods to assess sensitivity to benthic impacts suggest that use of multiple methods is more informative than a single method would be. For example, as the study area was relatively pristine we expected that a method associated with changes in abundance would be most successful as abundance could be expected to drop before taxa disappeared (Hewitt et al. 2005) and indeed the weighted abundance method could detect smaller impacts than the number of sensitive taxa. Conversely, in stressed areas, it could be expected that these two methods would show similar results and even that, in highly stressed areas, the number of highly sensitive taxa may be more useful. This could occur even if sensitive taxa were rare, as the number of rare taxa has been shown to decrease with stress (Gray 1997; Jackson 2001; Hewitt et al. 2009). In our results, the sensitive relative abundance method varied widely in its ability to detect impacts associated with different stressors. It was the best method for extraction but was not able to detect even a $90 \%$ reduction in the abundance of sensitive taxa for suspended sediment. This suggests that the taxa sensitive to extraction occurred in the study area in generally lower abundances than those sensitive to suspended sediment and in communities of lower total abundances.

Deriving an area's sensitivity to a stressor based on BTA does not only depend on the method used (e.g., weighted abundance, number of sensitive taxa) and the previous stressors impacting on the site. Other factors likely to be important are the traits selected to express sensitivity and the knowledge of traits exhibited by particular species. The decision trees in this study are simplistic representations based on traits that are generally well known. Two studies (Hewitt et al. 2008; Hewitt et al. 2011) tested for the effect of uncertain knowledge of species biological traits on fuzzy coding, by randomly changing the fuzzy coding of $10 \%$ of uncertain traits and compared results with 
the original results. In both cases, the general results remained the same, probably for two reasons. Firstly, abundance-based methods are unlikely to be strongly affected as biological traits of abundant and common species are generally known. Secondly, while we know less about biological traits of rare taxa, the traits that commonly separate high or medium sensitivity from those which will be unaffected are generally among the more easily assessed traits (e.g., size, fragility, feeding mode).

More generally, with the increasing use of BTA and databases storing knowledge of traits (e.g., MarLIN 2006), it is probable that our decision rules could be improved upon. However, the rules we used, based on the ecological literature, are sufficient for testing the hypotheses of this study. In fact, the weightings used in the derivation of the weighted abundance are likely to be more important than the rules, in particular whether taxa that benefit should be used to counter adverse effects on other taxa. This does not imply a shortcoming of the results from this study, rather that the weightings should reflect the purpose of the study. Whether taxa that benefit should offset adverse effects on other taxa is a decision that should be made, and the rationale explicitly stated in each study, at least until a consensus is reached through a variety of studies in different locations and with different stressors. The influence of the number of weighting categories on the ability of the methods to detect significant decreases could also be a useful aspect to explore in future research.

Increasingly in conservation and management there is a need to identify areas or habitats that may be especially at risk from different stressors, e.g., Ecologically or Biologically Significant Areas (Clark et al 2014) and vulnerable marine ecosystems (Auster et al. 2011). Ecosystem-based management, including spatial planning exercises, need to balance expected outcomes of different activities and require metrics and systematic methods of assessing sensitivity (or likelihood of impact) in order to achieve this (Williams et al. 2009; Clark et al. 2016). The results of this study add to the evidence that biological trait analysis offers a relatively simple and cost-effective way to develop robust 
metrics to assess sensitivities of areas, particularly if more than one method/metric is used. With the collection of more information on the traits held by species and by linking specific traits to ecosystem functions, biological trait analysis also offers those involved in ecosystem-based management the ability to go beyond assessing sensitivity to assess effects on ecosystem functioning, services and values.

\section{Supporting Information}

Map of sampling location (Appendix S1), information on taxa sensitivity (Appendix S2), a description of the taxa characteristic of each simprof group (Appendix S3) and a plot of the mean and standard errors of each simprof group for the different stressors and methods (Appendix S4) are available online. Also available are two example Moran's I autocorrelograms (Appendix S5). The authors are solely responsible for the content and functionality of these materials. Queries (other than absence of the material) should be directed to the corresponding author.

\section{Acknowledgements}

The data analysed in this manuscript was provided by Chatham Rock Phosphate Ltd. Funding for this work was provided by two sources: Strategic Science Investment Funding of the Coasts and Oceans Centre (New Zealand National Institute of Water and Atmospheric Research) project COME1701; and the New Zealand Ministry of Business, Innovation and Employment (New Zealand's major source of public good research funding) project "Enabling Management of Offshore Mining" led by Dr Malcolm Clark NIWA. We also thank Malcolm for commenting on a draft of this manuscript.

\section{Literature cited}

Aubin I, et al. 2016. Traits to stay, traits to move: a review of functional traits to assess sensitivity and adaptive capacity of temperate and boreal trees to climate change. Environmental Reviews 24:164-186.

Auster PJ, Gjerde KM, Heupel E, Watling L, Grehan A, Rogers AD. 2011. Definition and detection of vulnerable marine ecosystems on the high seas: problems with the "move-on" rule. ICES Journal of Marine Science 68:254-264.

This article is protected by copyright. All rights reserved. 
Bolam SG, Fernandes TF, Huxham M. 2002. Diversity, biomass and ecosystem processes in the marine benthos. Ecological Monographs 72:519-616.

Boschen RE, Rowden AA, Clark MR, Pallentin A, Gardner JP. 2016. Seafloor massive sulfide deposits support unique megafaunal assemblages: Implications for seabed mining and conservation. Marine environmental research 115:78-88.

Bremner J, Rogers SI, Frid CLJ. 2006. Matching biological traits to environmental conditions in marine benthic ecosystems. Journal of Marine Systems 60:302-316.

Clark MR, Althaus F, Schlacher TA, Williams A, Bowden DA, Rowden AA. 2016. The impacts of deepsea fisheries on benthic communities: a review. ICES Journal of Marine Science: Journal du Conseil 73:i51-i69.

Clark MR, Rowden AA, Schlacher TA, Guinotte J, Dunstan PK, Williams A, O'Hara TD, Watling L, Niklitschek E, Tsuchida S. 2014. Identifying Ecologically or Biologically Significant Areas (EBSA): A systematic method and its application to seamounts in the South Pacific Ocean. Ocean \& Coastal Management 91:65-79.

de Juan S, Demestre M. 2012. A Trawl Disturbance Indicator to quantify large scale fishing impact on benthic ecosystems. Ecological Indicators 18:183-190.

de Juan S, Demestre M, Thrush SF. 2009. Defining ecological indicators of trawling disturbance when everywhere that can be fished is fished: a Mediterranean case study. Marine Policy 33:472478.

Delgado-Baquerizo M, Giaramida L, Reich PB, Khachane AN, Hamonts K, Edwards C, Lawton LA, Singh BK. 2016. Lack of functional redundancy in the relationship between microbial diversity and ecosystem functioning. Journal of Ecology.

Ellis J, Cummings V, Hewitt J, Thrush S, Norkko A. 2002. Determining effects of suspended sediment on condition of a suspension feeding bivalve (Atrina zelandica): results of a survey, a laboratory experiment and a field transplant experiment. Journal of Experimental Marine Biology and Ecology 267:147-174.

This article is protected by copyright. All rights reserved. 
Emmerson M, Raffaelli D. 2000. Detecting the effects of diversity on measures of ecosystem function: experimental design, null models and empirical observations. Oikos:195-203.

Gray JS. 1997. Marine biodiversity: patterns, threats and conservation needs. Biodiversity and Conservation 6:153-175.

Hare JA, et al. 2016. A Vulnerability Assessment of Fish and Invertebrates to Climate Change on the Northeast U.S. Continental Shelf. PLoS ONE 11:e0146756.

Harvey E, Séguin A, Nozais C, Archambault P, Gravel D. 2013. Identity effects dominate the impacts of multiple species extinctions on the functioning of complex food webs. Ecology 94:169179.

Hewitt J, Julian K, Bone EK 2011. Chatham-Challenger Ocean Survey 20/20 Post-Voyage Analyses: Objective 10 - Biotic habitats and their sensitivity to physical disturbance. Ministry for Primary Industries, Wellington.

Hewitt JE, Anderson MJ, Hickey C, Kelly S, Thrush SF. 2009. Enhancing the ecological significance of contamination guidelines through integration with community analysis. Environmental Science and Technology 43:2118-2123.

Hewitt JE, Anderson MJ, Thrush SF. 2005. Assessing and monitoring ecological community health in marine systems. Ecological Applications 15:942-953.

Hewitt JE, Thrush SF, Dayton PD. 2008. Habitat variation, species diversity and ecological functioning in a marine system. Journal of Experimental Marine Biology and Ecology 366:116-122.

Hiddink J, Jennings S, Kaiser M. 2007. Assessing and predicting the relative ecological impacts of disturbance on habitats with different sensitivities. Journal of Applied Ecology 44:405-413. Hobday AJ, et al. 2011. Ecological risk assessment for the effects of fishing. Fisheries Research 108:372-384.

Hodgson D, McDonald JL, Hosken DJ. 2015. What do you mean, 'resilient'? . Trends in Ecology \& Evolution 30:503-506.

This article is protected by copyright. All rights reserved. 
Jackson JBC. 2001. What is natural in the coastal oceans? Proceedings of the National Academy of Science 98:5411-5418.

Lambert GI, Jennings S, Kaiser MJ, Davies TW, Hiddink JG. 2014. Quantifying recovery rates and resilience of seabed habitats impacted by bottom fishing. Journal of Applied Ecology 51:1326-1336.

Lavorel S, Grigulis K, Lamarque P, Colace MP, Garden D, Girel J, Pellet G, Douzet R. 2011. Using plant functional traits to understand the landscape distribution of multiple ecosystem services. Journal of Ecology 99:135-147.

MarLIN. 2006. BIOTIC - Biological Traits Information Catalogue. . Marine Life Information Network. Marine Biological Association of the United Kingdom. [Cited insert date] Plymouth.

McGill BJ, Enquist BJ, Weiher E, Westoby M. 2006. Rebuilding community ecology from functional traits. Trends in Ecology \& Evolution 21:178-185.

Menezes S, Baird DJ, Soares AMVM. 2010. Beyond taxonomy: a review of macroinvertebrate traitbased community descriptors as tools for freshwater biomonitoring. Journal of Applied Ecology 47:711-719.

Oug E, Fleddum A, Rygg B, Olsgard F. 2012. Biological traits analyses in the study of pollution gradients and ecological functioning of marine soft bottom species assemblages in a fjord ecosystem. Journal of Experimental Marine Biology and Ecology 432:94-105.

Pearson TH, Rosenberg R. 1978. Macrobenthic succession in relation to organic enrichment and pollution of the marine environment. Oceanography and Marine Biology: an Annual Review 16:229-311.

Rangel TF, Diniz-Filho JAF, Bini LM. 2010. SAM: a comprehensive application for spatial analysis in macroecology. Ecography 33:46-50.

Ribeiro DC, Costa S, Guilhermino L. 2016. A framework to assess the vulnerability of estuarine systems for use in ecological risk assessment. Ocean \& Coastal Management 119:267-277. 
Ricotta C, Acosta A. 2014. On the functional diversity of partially distinct species: some theory and a practical example. Community Ecology 15:205-211.

Rijnsdorp AD, et al. 2015. Towards a framework for the quantitative assessment of trawling impact on the seabed and benthic ecosystem. ICES Journal of Marine Science: Journal du Conseil.

SAS/STAT. 2011. SAS/STAT software, Version 9.3 of the SAS System for Microsoft. Copyright ( 20022011. SAS Institute Inc., Cary, North Carolina, U.S.A.

Sirot C, Villéger S, Mouillot D, Darnaude AM, Ramos-Miranda J, Flores-Hernandez D, Panfili J. 2015. Combinations of biological attributes predict temporal dynamics of fish species in response to environmental changes. Ecological Indicators 48:147-156.

Stark JD, Maxted JR. 2007. A biotic index for New Zealand's soft-bottomed streams. New Zealand Journal of Marine and Freshwater Research 41:43-61.

Strong JA, Andonegi E, Bizsel KC, Danovaro R, Elliott M, Franco A, Garces E, Little S, Mazik K, Moncheva S. 2015. Marine biodiversity and ecosystem function relationships: the potential for practical monitoring applications. Estuarine, Coastal and Shelf Science 161:46-64.

Thrush SF, Dayton PK. 2002. Disturbance to marine benthic habitats by trawling and dredging Implications for marine biodiversity. Annual Review of Ecology and Systematics 33:449-473.

Thrush SF, Hewitt JE, Cummings VJ, Dayton PK. 1995. The impact of habitat disturbance by scallop dredging on marine benthic communities: what can be predicted from the results of experiments? Marine Ecology Progress Series 129:141-150.

Thrush SF, Hewitt JE, Cummings VJ, Ellis JI, Hatton C, Lohrer A, Norkko A. 2004. Muddy waters: elevating sediment input to coastal and estuarine habitats. Frontiers in Ecology and Environment 2:299-306.

Thrush SF, Hewitt JE, Dayton PK, Coco G, Lohrer AM, Norkko A, Norkko J, Chiantore M. 2009. Forecasting the limits of resilience: integrating empirical research with theory Proceedings of the Royal Society B 276:3209-3217.

This article is protected by copyright. All rights reserved. 
Tillin HM, Hiddink J, Jennings S, Kaiser M. 2006. Chronic bottom trawling alters the functional composition of benthic invertebrate communities on a sea-basin scale. Marine Ecology Progress Series 318:31-45.

Tolonen KE, Tokola L, Grönroos M, Hjort J, Kärnä O-M, Erkinaro J, Heino J. 2016. Hierarchical decomposition of trait patterns of macroinvertebrate communities in subarctic streams. Freshwater Science 35:1032-1048.

Tyler-Walters H, Rogers SI, Marshall CE, Hiscock K. 2009. A method to assess the sensitivity of sedimentary communities to fishing activities. Aquatic Conservation: Marine and Freshwater Ecosystems 19:285-300.

Williams A, Gardner C, Althaus F, Barker B, Mills D. 2009. Understanding shelf-break habitat for sustainable management of fisheries with spatial overlap. Final report to the FRDC, project:250.

Williams SE, Shoo LP, Isaac JL, Hoffmann AA, Langham G. 2008. Towards an integrated framework for assessing the vulnerability of species to climate change. PLoS Biol 6:e325.

Zacharias MA, Gregr EJ. 2005. Sensitivity and vulnerability in marine environments: an approach to identifying vulnerable marine areas. Conservation Biology 19:86-97.

Zhang J-T, Fan L, Li M. 2012. Functional diversity in plant communities: theory and analysis methods. African Journal of Biotechnology 11:1014-1022. 
Table 1: Trait categories used in the study and their potential expressions.
Trait category
Expression

Adult movement

*Sedentary, Swimmer, *Crawler, Rafter/Drifter/Byssus, *Slow

burrower, *Fast burrower

Living position

Deep (>5 cm), *Middle (between 2-5 cm), *Top (in top $2 \mathrm{~cm}$ ),

*Epibenthic, *Bentho-pelagic

Feeding mode

*Suspension/filter, *Sub-surface deposit, *Surface deposit,

*Predator, *Scavenger, *Herbivore, Miner/Borer, *Photo

synthesisers, Photosynthetic endosymbionts, Chemosynthetic

(bacteria in tissues), Parasite

Growth form above surface $\quad *$ None, *Tubes, *Branching, *Erect, *Encrusting

Fragility

*Highly breakable, *Limited flexibility, *Extremely flexible

Size

*0-10 mm, *11-20 mm, 21-50 mm, 51-100 mm, >100 mm

* Trait expressions used in Fig. 1 and Appendices S1 and S2.

This article is protected by copyright. All rights reserved. 
Table 2: Number of transects sampled in each simprof group together with biological and environmental information (average, standard deviations) for those groups with $>2$ transects sampled.

$\begin{array}{lllllll}\text { SIMPROF } & \text { Number of } & \text { Total } & \text { Total } & \text { Depth }(\mathrm{m}) & \text { Slope } & \text { Mud (\% } \\ \text { group } & \text { transects } & \text { abundance } & \text { number of } & \text { weight) } \\ & & \left(\mathrm{m}^{2}\right) & \text { taxa } & \\ & & \text { (transect) } & \end{array}$

\begin{tabular}{lllllll}
\hline a & 11 & $16.9,7.6$ & $7.3,3.0$ & $392.4,46.6$ & $1.3,0.8$ & $32.3,9.1$ \\
b & 4 & $23.0,18.4$ & $2.8,0.5$ & $411.9,42.7$ & $1.0,0.4$ & $25.8,11.1$
\end{tabular}

$c$

2

8

$137.3,85.8 \quad 15.8,2.3$

$376.3,37.4$

$1.7,0.8$

$41.8,5.6$

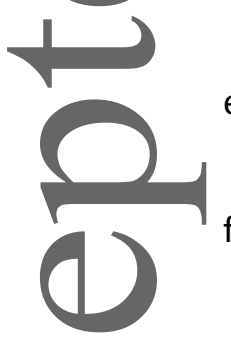

e

2

2

1

1

2

13

$153.3,35.1$

$13.8,2.5$

$399.0,13.5 \quad 1.5,0.5$

$29.4,11.2$

k

2

I

21

$388.3,147.9 \quad 21.7,3.3$

$396.8,9.8$

$2.1,0.9$

$33.4,16.3$ 


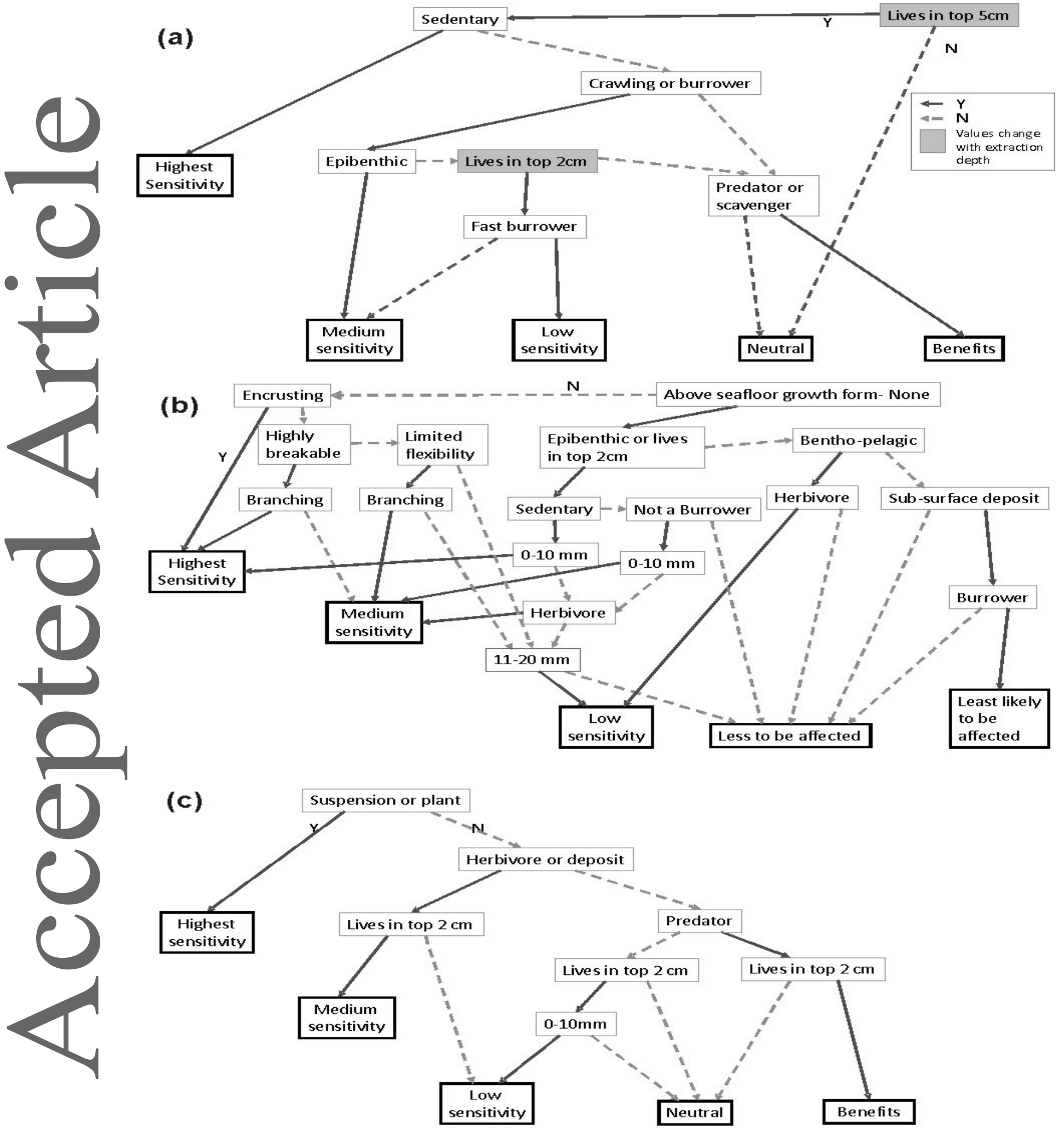

Figure 1: Decision trees used to assign a taxon's level of sensitivity, based on its biological traits, to (a) an extraction method penetrating to $5 \mathrm{~cm}$ deep, (b) sedimentation and (c) suspended sediment. . Information on trait categories is given in Table 1. Solid lines follow an answer "yes" to the trait expression question, dashed lines follow "no" answers. 


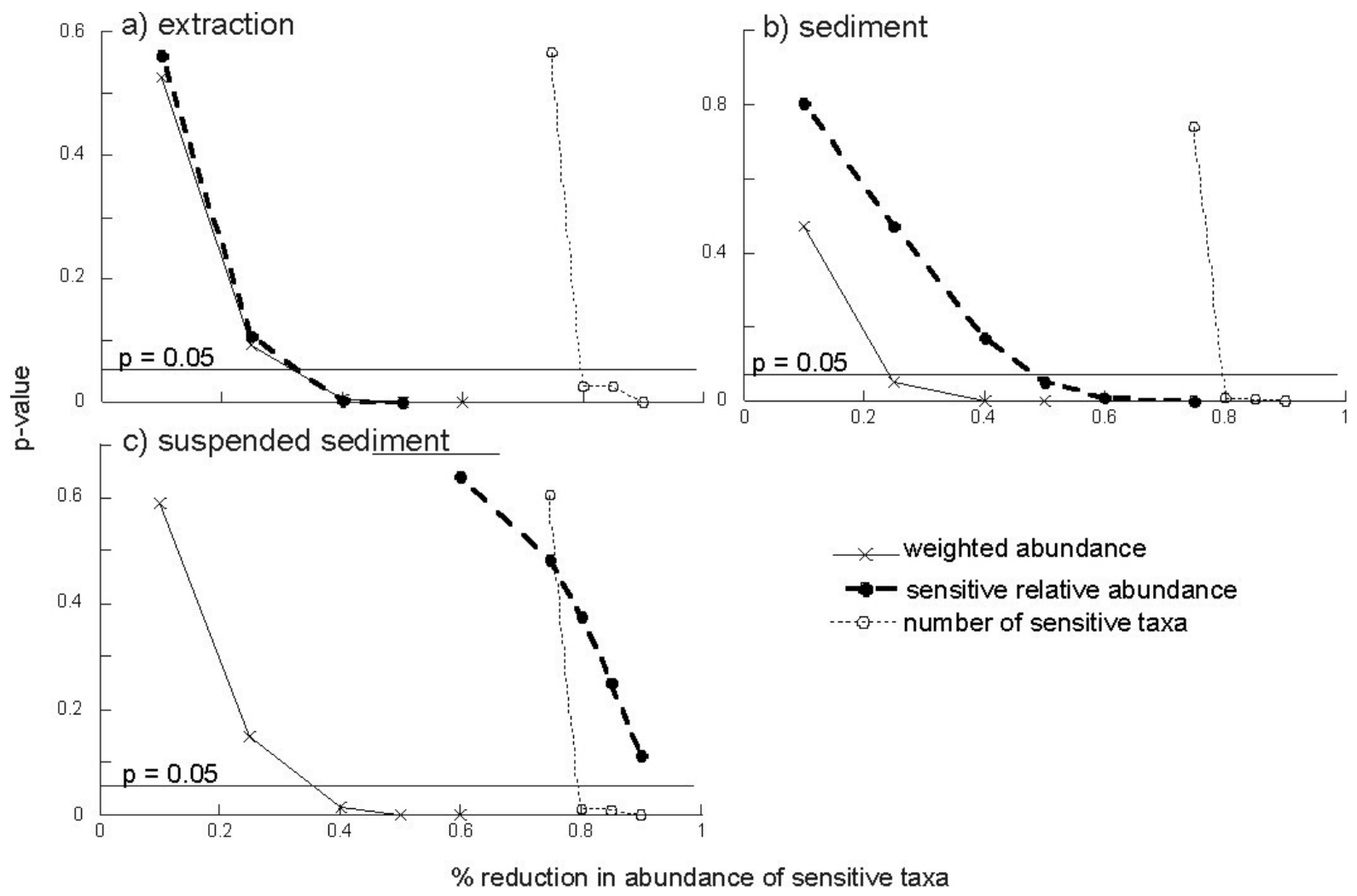

Figure 2: Decreasing $p$-values associated with t-tests of differences between the means of the original data and data with simulated reductions.

This article is protected by copyright. All rights reserved. 